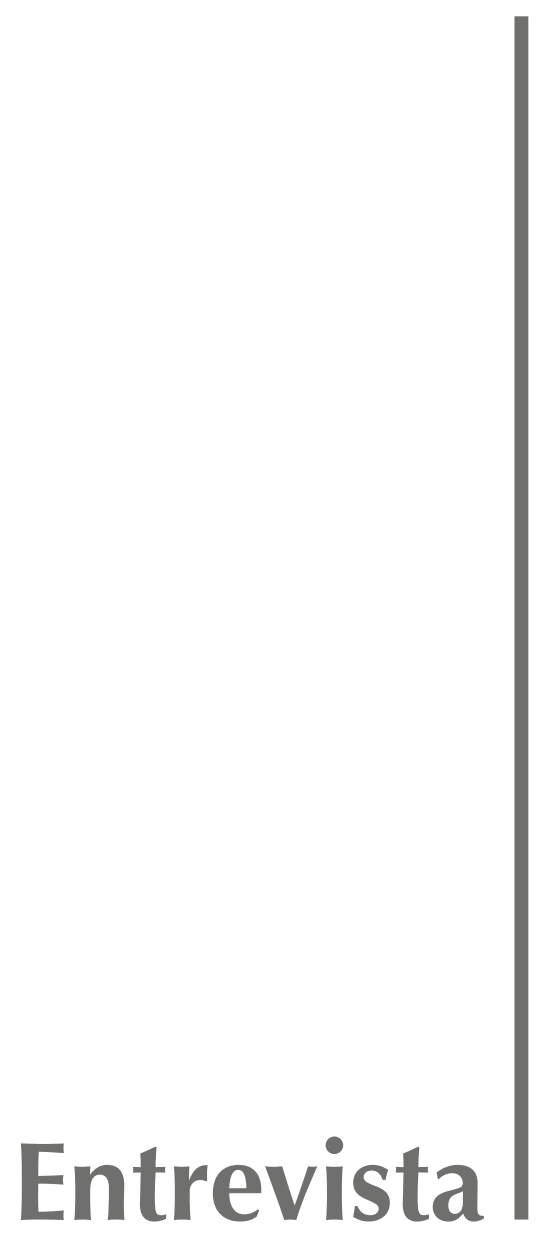





\title{
Entrevista a Aoife Nolan: "Recientes avances en el reconocimiento del derecho del niño y niña a ser oídos en los procedimientos internacionales" ${ }^{*}$
}

\author{
Por Ona Flores
}

\begin{abstract}
Ona Flores es abogada, egresada de la Universidad Católica Andrés Bello, Caracas, Venezuela y Máster en Derecho (LL.M.) de la Universidad de Columbia, N.Y. (Harlan Fiske Stone Scholar y Fulbright Fellow). Ha sido Investigadora del Programa de Mujeres, Género y Derechos Humanos del Centro de Derechos Humanos de la Universidad de Chile (2011-2013) y abogada senior de la Secretaría de la Corte Interamericana de Derechos Humanos (2003-2010).
\end{abstract}

Aoife Nolan es profesora de Derecho Internacional de los Derechos Humanos de la Facultad de Derecho de la Universidad de Nottingham. Se graduó con un LL.B de Trinity College Dublin en 2000 y completó su PhD en European University Institute, Florencia, en 2005. Ha publicado profusamente, en particular sobre materias relacionadas con derechos económicos, sociales y culturales, derechos de los niños y niñas así como también en derecho constitucional. Su trabajo ha sido publicado en prestigiosas revistas incluyendo Public Law, Human Rights Law Review y European Human Rights Law Review. Su libro Children's Socio-economic Rights, Democracy and the Courts fue publicado por Hart Publishing en septiembre de 2011. Obtuvo el IALT Kevin Boyle Book Prize y fue preseleccionada en Birks Book Prize. Su trabajo actual está enfocado en los derechos económicos, sociales y culturales después de las crisis económicas y financieras.

\section{Introducción}

El artículo 12 de la Convención sobre los Derechos del Niño (CDN) establece el derecho de los niños y niñas a expresar sus opiniones de manera libre en todos aquellos asuntos que los afecten y a que esas opiniones sean tomadas debidamente en cuenta. Asimismo, reconoce el derecho de todo niño a que se le ofrezca la posibilidad de ser escuchado en todo procedimiento judicial o administrativo que lo afecte ${ }^{1}$.

Según el Comité de los Derechos del Niño este artículo constituye uno de los valores fundamentales de la $\mathrm{CDN}^{2}$. Al interpretar otros tratados, varios organismos internacionales y regionales de

\footnotetext{
* Entrevista traducida del inglés por Alia Trabucco Zerán.

1 "1. Los Estados Partes garantizarán al niño que esté en condiciones de formarse un juicio propio el derecho de expresar su opinión libremente en todos los asuntos que afectan al niño, teniéndose debidamente en cuenta las opiniones del niño, en función de la edad y madurez del niño. 2. Con tal fin, se dará en particular al niño oportunidad de ser escuchado, en todo procedimiento judicial o administrativo que afecte al niño, ya sea directamente o por medio de un representante o de un órgano apropiado, en consonancia con las normas de procedimiento de la ley nacional". ONU. Convención sobre los Derechos del Niño. Adoptada el 20 de noviembre de 1989, artículo 12.

2 Comité de los Derechos del Niño. Observación General No. 12. El derecho a ser escuchado. 20 de julio de 2009. CRC/C/GC/12, párr. 2.
} 
derechos humanos también han reconocido el rol clave de este derecho en el marco de la CDN y su importancia en el ejercicio de otros derechos por parte de los niños y niñas ${ }^{3}$.

En la última década la mayoría de los dictámenes y recomendaciones adoptados por organismos internacionales de derechos humanos han estado dirigidos a impulsar cambios en la legislación y práctica de los Estados para que éstos garanticen el derecho de niños y niñas a ser oídos en procedimientos judiciales y administrativos internos. En contraste, la necesidad de adecuar los procedimientos internacionales a las necesidades y particularidades propias de los niños y niñas, de conformidad con el artículo 12 de la CDN, forma parte de una nueva discusión que apenas comienza a ser reconocida.

Un caso emblemático en esta materia fue decidido por la Corte Interamericana de Derechos Humanos en el año 2012. La decisión de la Corte Interamericana en el caso Atala Riffo e Hijas vs. Chile, relativo a la discriminación por orientación sexual en procedimientos de custodia infantil, abordó por primera vez la cuestión de la participación y representación de los niños y niñas en un procedimiento contencioso internacional.

Pocos meses antes de alcanzar una decisión, la Corte emitió una resolución señalando que "en ninguna parte del expediente había una manifestación precisa por parte de las niñas M., V. y R. respecto a si estaban de acuerdo con la representación que ejercía cualquiera de sus padres y de si deseaban ser consideradas como presuntas víctimas en el presente caso" ${ }^{\prime 4}$. La Corte indicó que "si bien existían dos escritos mediante los cuales tanto el padre como la madre manifestaban que actuaban en representación de las tres niñas ante este Tribunal, la posición de la madre y el padre no necesariamente representaban los intereses de las niñas" ${ }^{\prime \prime}$. Como medida para mejor resolver, la Corte tomó la decisión, sin precedentes, de enviar a personal de su Secretaría a Chile, para así ofrecer a las tres niñas la oportunidad de ser oídas en el procedimiento ante la Corte ${ }^{6}$.

La discusión en torno a la participación de las niñas en el caso Atala Riffo e Hijas refleja el debate surgido a propósito de la adopción del nuevo Protocolo Facultativo de la Convención sobre los Derechos del Niño relativo a un Procedimiento de Comunicaciones (PF3 CDN) -abierto

3 En particular, la Corte Interamericana de Derechos Humanos reconoció hace mucho que "[t]anto la Convención Americana como la Convención sobre los Derechos del Niño forman parte de un muy comprensivo corpus juris internacional de protección de los niños que debe servir a esta Corte para fijar el contenido y los alcances de la disposición general definida en el artículo 19 de la Convención Americana". Corte IDH. Caso de los "Niños de la Calle" (Villagrán Morales y otros) vs. Guatemala. Fondo. Sentencia de 19 de noviembre de 1999. Serie C No. 63, párr. 193.

4 Corte IDH, Caso Atala Riffo y Niñas vs. Chile. Fondo, Reparaciones y Costas. Sentencia del 24 de febrero de 2012. Serie C No. 239, párr. 67.

5 “Durante dicha diligencia, el personal de la Secretaría estuvo acompañado por la psiquiatra María Alicia Espinoza. Antes de realizar la diligencia, la delegación de la Secretaría de la Corte sostuvo una reunión previa con dicha psiquiatra, la cual consistió en un intercambio de ideas con el fin de garantizar que la información brindada fuera accesible y apropiada para las niñas. Teniendo en cuenta los estándares internacionales sobre el derecho de las niñas y los niños a ser oídos, las niñas M. y R. fueron, en primer lugar, informadas de manera conjunta por el personal de la Secretaría sobre su derecho a ser oídas, los efectos o consecuencias que podían producir sus opiniones dentro del proceso contencioso en el presente caso, la posición y los alegatos de las partes en el presente caso, y se les consultó si querían continuar participando en la diligencia. Posteriormente, en lugar de desarrollar un examen unilateral, se sostuvo una conversación con cada niña por separado, con el objetivo de brindar un ambiente propicio y de confianza a las niñas. Durante la diligencia no estuvieron presentes ninguno de los padres y ninguna de las partes. Además, la diligencia realizada con las niñas fue privada en razón del pedido de confidencialidad de la identidad de las niñas que han realizado tanto la Comisión como los representantes en el presente caso [...] como por la necesidad de proteger el interés superior de las niñas y su derecho a la intimidad. Además, las niñas solicitaron expresamente que se mantuviera absoluta reserva de todo lo que manifestaran en la reunión". Ibídem, párr. 67.

6 Ibídem, párr. 68. 
oficialmente a firma y ratificación en febrero de 2012-7. Durante los tres años que le llevó al Grupo de Trabajo Abierto elaborar el Protocolo Facultativo 3, gran parte de la discusión se centró en el tipo de procedimientos que serían necesarios para garantizar alivio sustantivo y procesal en los casos de violaciones a los derechos humanos de los niños. La idea de que el nuevo procedimiento debería garantizar que los niños y niñas pudiesen interponer peticiones directamente ante el Comité y potenciar su agencia fue el eje de todas las recomendaciones y de las opiniones de expertos recibidas por el Grupo de Trabajo Abierto.

Tanto la decisión de la Corte Interamericana en el caso Atala Riffo e hijas vs. Chile como la adopción del Protocolo Facultativo 3 ponen de relieve la necesidad de que los organismos internacionales cambien y adopten nuevas prácticas para ajustarse a los derechos de los niños y niñas a ser escuchados según el artículo 12 de la CDN y facilitar su acceso a los mecanismos de quejas. La entrevista con la profesora Aoife Nolan buscó abordar los aspectos más relevantes de esta discusión y conocer su opinión sobre los avances en esta materia acontecidos en 2012.

\section{Ona Flores: ¿Cómo ha interpretado el Comité de los Derechos del Niño el artículo 12 de la Convención y, más específicamente, cuál ha sido su aproximación respecto del derecho de los niños y niñas a ser escuchados en los procedimientos judiciales?}

Aoife Nolan: El Comité de los Derechos del Niño ha puesto un fuerte énfasis en el desarrollo del artículo 12 de la Convención. En su Observación General No. 5 sobre Medidas Generales de Aplicación de la Convención sobre los Derechos del Niño, el Comité dejó en claro que esta disposición constituía uno de los cuatro Principios Generales de la CDN; es decir, es un principio clave que "se aplica por igual a todas las medidas adoptadas por los Estados para implementar la Convención" 8 .

La declaración más importante del Comité sobre el artículo 12 es su Observación General No. 12 sobre el Derecho del Niño a ser escuchado, en la que explica cómo debe hacerse efectivo este derecho en una serie de diferentes contextos, incluyendo la esfera de la salud, la educación y la familia. ¿Cómo ha interpretado el Comité este derecho? Como era de esperarse, considerando la redacción del artículo 12, el Comité ha dejado en claro en su Observación que el derecho establecido en este artículo se aplica solamente a niños que son 'capaces de formarse sus propias opiniones'. Además, si bien el niño tiene derecho a expresar esas opiniones libremente en todos aquellos asuntos que lo afecten, dichas opiniones no actúan como una "carta de triunfo" que anula todos los otros factores, ni actúa como elemento determinante en toda toma de decisiones que involucre al niño. Más bien, deben tomarse debidamente en cuenta las opiniones del niño en función de su edad y madurez. Un importante elemento a considerar es que ni el Comité sobre los Derechos del Niño ni la propia Convención establece una lista exhaustiva de reglas absolutas y rígidas respecto de (i) cómo debe ser garantizada la participación del niño en cada caso, ni (ii) qué tipo de actividades de consulta o participación deben ser implementadas en cada contexto de toma de decisiones.

7 Ver LANGFORD, Malcolm y CLARK, Sevda. The New Kid on The Block: A Complaints Procedure for the Convention on the Rights of the Child. Working Paper No. 1. Noruega: Socio Economic Rights Programme, Norwegian Centre for Human Rights, University of Oslo, 2010 y NEWELL, Peter. Submission to Open-ended Working Group of the Human Rights Council, considering the possibility of elaborating an Optional Protocol to provide a communications procedure for the Convention on the Rights of the Child. Ginebra, 14-18 de diciembre de 2009.

8 Comité de los Derechos del Niño. Observación General No. 5. Medidas generales de aplicación de la Convención sobre los Derechos del Niño (artículos 4 y 42 y párrafo 6 del artículo 44). 27 de noviembre de 2003. CRC/GC/2003/5, párr. 12. 
El artículo 12(2) establece que "se dará en particular al niño oportunidad de ser escuchado, en todo procedimiento judicial o administrativo que afecte al niño, ya sea directamente o por medio de un representante o de un órgano apropiado, en consonancia con las normas de procedimiento de la ley nacional". Si bien, a primera vista, esta parecería ser una poderosa directriz que otorga al niño el derecho a expresar sus opiniones en todo procedimiento judicial o administrativo que lo afecte, esto no es exactamente lo que establece el artículo 12(2). La última parte de dicho artículo puede ser motivo de preocupación, ya que la referencia a "las normas de procedimiento de la ley nacional" parecería sugerir que las reglas procedimentales del derecho interno podrían servir como una legítima limitación al grado en que los Estados estarían obligados a hacer efectivo el artículo 12(2). Esto dicho, el Comité ha subrayado que "no debe interpretarse que estos términos permiten utilizar legislación de procedimiento que restrinja o impida el disfrute de este derecho fundamental"9. Por lo tanto, el Comité interpreta el artículo 12(2) como un emplazamiento a los Estados a que den cumplimiento a las reglas básicas de un procedimiento justo. En términos de alcance, el Comité ha dejado en claro que el derecho a ser oído se aplica tanto a procedimientos judiciales como administrativos que sean iniciados por el niño y ha puesto énfasis en la importancia de que estos procedimientos sean accesibles y apropiados para el niño.

Ona Flores: Se ha señalado que la implementación del artículo 12 en los últimos años ha gatillado muchos cambios en los sistemas jurídicos nacionales y en la práctica judicial. ¿Estás de acuerdo con esta afirmación? ¿Has observado en tu trabajo de investigación mejores prácticas judiciales en relación con la participación de los niños en la determinación de sus derechos?

Aoife Nolan: Ciertamente estoy de acuerdo. Estos cambios han sido especialmente llamativos en el contexto del derecho de familia, los procedimientos de protección y cuidado del niño y la justicia de menores. Pero también son evidentes respecto de otros procedimientos legales, tales como los relativos a materias de inmigración y atención médica. Diversas jurisdicciones han intentado tomar en cuenta las opiniones del niño en una serie de procedimientos -aunque no siempre lo han hecho otorgando a los niños un papel directo o una "voz" en los procedimientos-. Los esfuerzos por hacer efectivo el artículo 12 se pueden observar en la utilización de una serie de diferentes mecanismos. Estos incluyen diversos medios a través de los cuales las opiniones de los niños pueden ser consideradas directamente en los procedimientos (por ejemplo, a través del suministro de evidencia por medio de videos de testimonios o de entrevistas judiciales a los niños), así como también a través de diferentes modelos de representación (por ejemplo, el uso de tutores o curadores legales, el nombramiento de "representantes de los niños" y el encargo de informes de peritos o de familia que otorguen información a la corte sobre las opiniones de los niños). Habiendo dicho esto, aún hay importantes problemas en la mayoría (si no en todas) de las jurisdicciones respecto de cómo garantizar que las opiniones de los niños -especialmente las opiniones de niños más pequeñossean tomadas debidamente en cuenta en los procedimientos judiciales.

\section{Ona Flores: ¿Cuáles son los valores y los objetivos que subyacen al artículo 12 y qué implicancias tienen para el concepto y el papel de los niños y niñas en la sociedad?}

Aoife Nolan: El artículo 12 ha sido descrito como el elemento más innovador de la CDN. Le interesa, en última instancia, reconocer la capacidad de agencia del niño sea al interior de la familia, la comunidad, la sociedad o en el contexto democrático. Reconoce la autonomía (real y potencial) del niño y busca hacerla efectiva en los diversos procedimientos de toma de decisiones

9 Comité de los Derechos del Niño. Observación General No. 12. op. cit., párr. 38 
que afecten a los niños y a sus derechos. Tradicionalmente, los niños eran vistos como sujetos de patria potestad. En contraste, la CDN considera a los niños como titulares de derechos por derecho propio. El artículo 12 conceptualiza a los niños como actores, no sólo como seres pasivos.

Se ha argumentado que, potencialmente, el artículo 12 podría entrar en conflicto con la obligación establecida en el artículo 3(1) de la CDN, que establece que el interés superior del niño debe ser una consideración primordial en todas las medidas que afecten a los niños. Uno podría argumentar que el artículo 3(1) es paternalista por naturaleza y que exige que el interés superior del niño "triunfe" sobre sus propias opiniones en aquellos casos en que ambas entren en conflicto. En contraste, el Comité ha señalado que "[n]o existe tensión entre los artículos 3 y 12, sino solamente complementariedad entre los dos principios generales: uno establece el objetivo de alcanzar el interés superior del niño y el otro ofrece la metodología para lograr el objetivo de escuchar al niño o a los niños. En realidad, no es posible una aplicación correcta del artículo 3, sino se respetan los componentes del artículo 12. Del mismo modo, el artículo 3 refuerza la funcionalidad del artículo 12 al facilitar el papel esencial de los niños en todas las decisiones que afecten a su vida"10. A pesar de esta declaración del Comité, en la práctica parecería existir un claro potencial de conflicto entre los artículos 12(1) y 3(1). Como es el caso de todos los derechos que están en potencial conflicto, el balance entre ellos debe ser determinado caso a caso. Sin embargo, es notable que para la CDN, tanto la determinación de cuál sería el interés superior del niño, como la decisión sobre el peso que debería otorgarse a las opiniones de los niños, quede reservada a una decisión tomada por adultos. De este modo, las opiniones de los niños están lejos de ser determinantes en el marco establecido por la CDN.

\section{Ona Flores: A la luz del avance en el desarrollo del contenido y alcance del artículo 12 de la CDN descrito, ¿cuál dirías que es el papel del niño en procedimientos de los organismos inter- nacionales y regionales de derechos humanos con funciones judiciales o cuasi judiciales?}

Aoife Nolan: Todas las personas, incluidos los niños, tienen derechos establecidos tanto en tratados de la ONU, como es el caso del Pacto Internacional de Derechos Civiles y Políticos ${ }^{11}$, como en instrumentos regionales de derechos humanos, como la Convención Americana sobre Derechos Humanos ${ }^{12}$, el Convenio Europeo para la Protección de los Derechos Humanos y de las Libertades Fundamentales ${ }^{13}$ y la Carta Africana sobre los Derechos Humanos y de los Pueblos ${ }^{14}$, y tienen el derecho a exigir el cumplimiento de tales derechos sin importar su estatus de niño o de adulto. Sin embargo, los niños rara vez interponen peticiones directas en los procedimientos internacionales o regionales. En lugar de ello, en muchos casos, las demandas son interpuestas en su nombre por sus padres u otros custodios. Esto se encuentra expresamente contemplado en las disposiciones aplicables a diversos mecanismos de supervisión, que permiten que las peticiones sean interpuestas "a nombre de" las víctimas, siempre y cuando sean satisfechas ciertas condiciones ${ }^{15}$. Un

10 Ibídem, párr. 74.

11 Ver, ONU. Pacto Internacional de Derechos Civiles y Políticos. Adoptado el 16 de diciembre de 1966, artículo 2.

12 Ver, OEA. Convención Americana sobre Derechos Humanos. Adoptada el 22 de noviembre de 1969, artículo 1.

13 Ver, Convenio Europeo para la Protección de los Derechos Humanos y de las Libertades Fundamentales. Adoptado el 4 de noviembre de 1950, artículo 1.

14 Ver, Carta Africana sobre los Derechos Humanos y de los Pueblos. Adoptada el 27 de julio de 1981, artículo 2.

15 Ver, por ejemplo, artículo 3 PF-CDN; African Union. Lineamientos para la Consideración de las Comunicaciones Establecidas en el Artículo 44 de la Carta Africana sobre los Derechos y el Bienestar del Niño. ACERWC/8/4. [en línea] $<$ http://acerwc.org/wp-content/uploads/2011/03/ACERWC-Guidelines-on-Communications-English.pdf> [consulta: 15 abril 2013]. 
ejemplo dentro del sistema interamericano (SIDH) es la sentencia Yean y Bosico vs. República Dominicana, de la Corte Interamericana de Derechos Humanos ${ }^{16}$. El origen de este fallo fue una petición interpuesta por varias ONGs "como representantes de los peticionarios"17. La tendencia de que sean representantes adultos quienes interpongan demandas también puede verse en la Corte Europea de Derechos Humanos. El único organismo basado en un tratado de derechos humanos que se ocupa exclusivamente de los derechos del niño y que en la actualidad tiene un procedimiento de reclamaciones especialmente diseñado con este propósito -el Comité Africano de los Derechos y el Bienestar del Niño- hasta ahora ha completado una única comunicación. La petición fue presentada por una serie de ONGs en nombre de víctimas infantiles ${ }^{18}$.

La preocupación que surge de lo anterior es que si bien es cierto que los derechos del niño forman parte central de las decisiones que toman los organismos judiciales y cuasi judiciales a los que se presentan este tipo de casos, la prioridad que los niños podrían darles a cierto tipo de derechos no es necesariamente respetada cuando son los adultos los encargados de promover los derechos de los niños y no consultan adecuadamente su opinión sobre los temas en cuestión.

También resulta llamativo que incluso en aquellos casos en los que los derechos del niño han sido tenidos en cuenta por mecanismos de reclamación regionales e internacionales, los derechos de participación y autonomía no han recibido el mismo nivel de atención que, por ejemplo, los derechos relativos a la protección o provisión del niño ${ }^{19}$. No obstante, algunos aspectos de la participación han sido abordados hasta cierto punto; por ejemplo, la Corte Europea de Derechos Humanos ha abordado cuestiones relativas a la participación de los niños en casos de derecho al respeto de la vida privada, el derecho a la libertad de pensamiento, conciencia y religión, el derecho a la libre expresión y a la libertad de reunión y asociación.

Ona Flores: Creo que por esas razones, la decisión del caso Atala Riffo y Niñas vs. Chile resulta tan relevante para el avance del derecho de los niños y niñas a participar y ser escuchados en este tipo de procedimientos. En Chile, sin embargo, la sentencia fue polémica. Algunos cuestionaron su legitimidad en relación con las niñas. ¿En qué medida crees que la decisión de la Corte IDH satisfizo el artículo 12 de la CDN?

Aoife Nolan: La decisión de la Corte IDH sobre Atala Riffo satisfizo el estándar establecido por el artículo 12 de la CDN y por el Comité de los Derechos del Niño. En primer término, comparto la valoración que hace la Corte Interamericana de la vulneración de los derechos de las niñas a ser escuchadas y a que sus opiniones sean tenidas en cuenta en el sistema de justicia interno. El procedimiento ante el Tribunal de Menores de Villarrica (que incluyó una audiencia privada

16 Corte IDH. Caso de las Niñas Yean y Bosico vs. República Dominicana. Sentencia de 8 de septiembre de 2005. Serie C No. 130.

17 Ver, lbídem, párr. 6.

18 Comité Africano de los Derechos y el Bienestar del Niño. Institute for Human Rights \& Development in Africa and the Open Society Justice Initiative (on behalf of children of Nubian descent in Kenya) v Kenya. Communication No. 002/ Com/002/09. 22 de marzo de 2011.

19 Ver, por ejemplo, Corte IDH. Condición Jurídica y Derechos Humanos del Niño, Opinión Consultiva OC-17/02 del 28 de agosto de 2002. Serie A No. 17; Caso de los Hermanos Gómez-Paquiyauri vs. Peru. Fondo, Reparaciones y Costas. Sentencia del 8 de julio de 2004. Serie C No. 110; Caso del "Instituto de Reeducación del Menor" vs. Paraguay. Objeción Preliminar, Fondo, Reparaciones y Costas. Sentencia del 2 de septiembre de 2004. Serie C No. 112; Caso de las Hermanas Serrano-Cruz vs. El Salvador. Interpretación de la Sentencia de Fondo, Reparaciones y Costas. Sentencia del 9 de septiembre de 2005, Serie C No. 131. 
cuyo registro se guardó en una caja fuerte de la Corte $)^{20}$, y la expresa consideración prestada por el tribunal a la opinión de las niñas en su toma de decisiones, claramente se atuvieron al artículo 12. El argumento del Tribunal de Menores según el cual las declaraciones hechas por las niñas no influenciaron su decisión dada su corta edad y la posibilidad de que sus opiniones hubieran sido afectadas por factores externos, es potencialmente problemático en términos del artículo 12. Sin embargo, dado que el artículo 12 no exige que los niños tengan la "última palabra" en una toma de decisiones que los afecte, el tribunal estaba en libertad de decidir de este modo (aunque podríamos cuestionar si el tribunal tuvo debidamente en cuenta las opiniones de las niñas en función de su edad y madurez, dado que la mayor tenía ocho años cuando se realizó la audiencia en 2003). Considerando los hechos establecidos en la sentencia, resulta evidente que tal como falló la Corte Interamericana, la Corte de Apelaciones de Temuco y la Corte Suprema de Chile no garantizaron plenamente en su sentencia el derecho de los niños a ser oídos tal como lo estipula el artículo 12.

En este sentido, el veredicto de la Corte estipulando que el derecho del niño a ser oído en el artículo 8(1) de la Convención Americana debe ser interpretado a la luz del artículo 1221, así como su extensa referencia al artículo 12 y la Observación General No. 12 del Comité22 son encomiables. En este caso, la Corte adoptó una interpretación del artículo 8(1) centrada en el niño y procedió a aplicarla de manera efectiva en los procedimientos judiciales internos que condujeron al sistema interamericano ${ }^{23}$.

En segundo término, la parte de la sentencia de la Corte Interamericana que se enfoca en la "consideración previa sobre la participación de las niñas M., V. y R". ${ }^{24}$ en el procedimiento de justicia interamericano refleja una clara preocupación por parte de la Corte de que la opinión del niño sea oída y un claro avance en la materia. Es sorprendente que la Corte subrayara la ausencia de declaraciones por parte de las niñas sobre si estaban de acuerdo con la representación de sus respectivos padres o si deseaban ser consideradas como presuntas víctimas para los efectos del caso ante el SIDH. La declaración de la Corte acerca de que "los niños ejercen sus derechos de manera progresiva, a medida que desarrollan un mayor grado de independencia" demuestra que está consciente de que el derecho de los niños a ser oídos y a que sus opiniones sean tenidas en cuenta en la toma de decisiones que afecten sus derechos evoluciona a medida que los niños maduran y crecen. Las medidas dispuestas por la Corte en la audiencia efectuada en Chile (es decir, el hecho de que informara a las niñas sobre su derecho a ser oídas, así como también sus esfuerzos por adoptar un procedimiento adecuado a las necesidades de las niñas, de modo que pudiese garantizar que fueran informadas de su derecho y que se consultaran sus opiniones), al igual que el respeto y reconocimiento de la necesidad de proteger el interés superior de las niñas y su derecho a la privacidad, parecerían demostrar que se satisficieron los requerimientos del artículo 12. El fallo de la Corte sobre que "las dos niñas expresaron de manera libre e independiente sus propias opiniones y juicios formados sobre los hechos del caso que atañen a ellas, así

\footnotetext{
20 Corte IDH, Caso Atala Riffo y Niñas vs. Chile, op. cit., párr. 36.

21 Ibídem, párr. 196.

22 Ibídem, párrs. 197-198.

23 Ibídem, párrs. 202-208.

24 Ibídem, párrs. 67-71.
} 
como algunas de sus expectativas e intereses en la resolución del presente caso", y su decisión de considerarlas como presuntas víctimas también se ajusta al artículo $12^{25}$.

En general, la Corte debe ser elogiada por el modo en que utilizó esta decisión para ampliar su jurisprudencia previa sobre el derecho del niño a ser escuchado ${ }^{26}$. Si bien la declaración clave de la Corte sobre los derechos del niño se centra principalmente en el derecho del niño a ser oído en el contexto de la justicia de menores ${ }^{27}$, el caso Atala Riffo demuestra de qué manera el derecho a ser oído puede operar en otros contextos, en particular en casos de derecho de familia. De manera positiva, la Corte también hace referencia al artículo 2 de la CDN y al tratamiento discriminatorio que sufrieron las niñas a partir de la orientación sexual de su madre ${ }^{28}$. Asimismo, en su toma de decisiones le adjudicó una gran importancia al interés superior de las niñas ${ }^{29}$.

\section{Ona Flores: ¿Hay algún aspecto de la sentencia que criticarías?}

Aoife Nolan: Considero que la discusión sobre el artículo 17 (derecho a una familia) y el artículo 11 (derecho a la vida privada y familiar) de la Convención Americana no se enfocó de manera central en los derechos del niño establecidos a este respecto en la CDN. Esto es así a pesar del hecho de que el fallo de custodia en cuestión tendría consecuencias directas para los niños y su disfrute de esos derechos. De este modo, la sentencia de la Corte podría haber ido más lejos en este aspecto.

Ona Flores: Leyendo la sentencia, es posible imaginar lo difícil que puede ser para una Corte internacional -tan alejada inclusive físicamente del lugar donde ocurren los hechos- decidir sobre la representación de niños y valorar las acusaciones de "conflictos de interés, manipulación y elaboración de agendas de litigación" por parte de los adultos. En tu reciente libro Children's Socio-economic Rights, Democracy and the Courts [Los derechos socioeconómicos de los niños, la democracia y las Cortes] señalas que esas cuestiones pueden ser "desventajas" en el uso o rol de las Cortes en la promoción y defensa de los derechos de los niños. ¿Podrías explicar tu posición y conectarlo a las dificultades propias del litigio ante Cortes internacionales?

Aoife Nolan: En mi libro sostengo que los niños experimentan tanto incapacidades reales como artificialmente creadas en su participación en procedimientos legales. Por ejemplo, los niños son con frecuencia excluidos de participar en los procedimientos judiciales debido a limitaciones establecidas respecto de su facultad de demandar directamente la satisfacción de sus derechos, como es el caso de diversos sistemas legales nacionales. En la práctica, tal como ocurre en el ámbito internacional, aquellos casos que impliquen la consideración de derechos de los niños serán con frecuencia interpuestos en su nombre, ubicando a los niños un paso más atrás de lo que estaría un adulto legalmente empoderado en relación al mismo procedimiento. Este "alejamiento" de los niños del procedimiento judicial disminuye su capacidad para supervisar a sus representantes legales, establecer su propia "agenda" de litigación o garantizar que el procedimiento sea entablado según su visión de aquello que implicaría la satisfacción de sus derechos. Tal vez uno

\footnotetext{
25 Ibídem, párr. 69. La Corte escuchó a dos de las niñas el 8 de febrero de 2012. Una de las niñas no participó en las audiencias por razones de fuerza mayor. La Corte ordenó que "para efectos de las reparaciones la autoridad nacional competente para la infancia deberá constatar en forma privada la opinión libre de la niña V. sobre si desea ser considerada parte lesionada". Ibídem, párr. 71.

26 Ver, Corte IDH. Condición Jurídica y Derechos Humanos del Niño, op. cit., párrs. 99-102.

27 Ibídem, párrs. 99-100.

28 Corte IDH, Caso Atala Riffo y Niñas vs. Chile, op. cit., párr. 151.

29 Ibídem, párrs. 109-111.
} 
de los más grandes desafíos en términos de la representación es la relación entre los niños y sus abogados. La incapacidad legal de los niños (y su frecuente incapacitación legal), sus limitadas posibilidades de instruir o supervisar a sus representantes legales, en conjunto con las diferencias sociales, culturales, psicológicas -y desde luego etarias- entre los niños y sus abogados, pueden provocar que la mediación de los abogados sea problemática respecto de las opiniones de los niños.

El problema de la representación y del establecimiento de una agenda de litigación puede verse exacerbado en el contexto judicial internacional y regional. En primer lugar, muchos de los mismos problemas de los sistemas nacionales estarán también presentes, como es el caso de la representación de los niños por parte de adultos/abogados. Más aún, debido a que los titulares de derechos (incluidos los niños y/o sus representantes) no están habilitados para presentar casos directamente ante todos los organismos judiciales regionales (por ejemplo, ante la Corte Interamericana de Derechos Humanos), puede haber un alejamiento aún mayor de los niños respecto de estos procedimientos judiciales y de toma de decisiones que en el caso de los procedimientos internos.

\section{Ona Flores: ¿Crees que el Protocolo Facultativo de la CDN relativo a un Procedimiento de Comunicaciones, abierto a firma y ratificación en 2012, aborda efectivamente esta preocupación?}

Aoife Nolan: Hasta ahora, este innovador Protocolo Facultativo ha sido ratificado por sólo tres países y se necesita la ratificación de diez países antes de que pueda entrar en vigencia. Ha habido grandes avances en lo relativo al mecanismo contemplado por el Protocolo Facultativo. Por ejemplo, el artículo 2 del PF3-CDN sobre los principios generales que guían las funciones del Comité exige que el Comité contemple los derechos y opiniones del niño. Esta disposición hace eco del artículo 12 de la CDN, donde se establece que las opiniones de los niños deben tenerse en cuenta en función de su edad y madurez. El artículo 3 del PF3-CDN señala que "tendrá en cuenta en particular el artículo 2 del presente Protocolo para garantizar que los procedimientos se adapten al niño". De este modo, existe una evidente preocupación por garantizar un enfoque centrado en el niño. Esto es desarrollado en el artículo 3(2), el cual deja en claro que el Comité incluirá en su reglamento salvaguardias para evitar que quienes actúen en nombre de niños los manipulen -por ejemplo, padres u otros que presenten demandas basadas en los derechos del niño, pero que en los hechos tengan otros objetivos-. Igualmente, el Comité puede negarse a examinar toda comunicación que en su opinión no redunde en el interés superior del niño. Se trata de salvaguardias muy importantes surgidas a partir de una preocupación actual y legítima en relación a la potencial manipulación, por parte de los padres u otros adultos, de los niños y de temas relacionados con sus derechos para promover su propia agenda. Un ejemplo podría ser el caso de padres que intenten utilizar el derecho del niño a la libertad de religión con el fin de fomentar la existencia de escuelas religiosas que permitan el castigo físico, dado que el Comité ha declarado que el castigo físico del niño en un contexto educativo está prohibido por la Convención ${ }^{30}$. Ésta sería una instancia clara en la que el Comité podría y debería negarse a considerar una petición sobre los derechos del niño, porque evidentemente iría en contra de los intereses superiores del niño y su derecho a no ser sometido a actos de violencia.

30 Para mayor información, ver Comité de los Derechos del Niño. Observación General No. 8. El derecho del niño a la protección contra los castigos corporales y otras formas de castigo crueles o degradantes. CRC/C/GC/8. 21 de agosto de 2006 y Observación General No. 13. Derecho del niño a no ser objeto de ninguna forma de violencia. CRC/C/GC/13. 18 de abril de 2011. 
Existen, sin embargo, algunas importantes falencias en el mecanismo de reclamación. Es innegable que el final del proceso de elaboración del PF3-CDN fue hecho con cierto apuro ${ }^{31}$. En una declaración luego de que el PF3-CDN fuera adoptado, la influyente red NGO Group de la CDN dijo que "condenaba" el hecho de que el "enfoque de la última ronda de negociaciones del nuevo Protocolo fuera lograr un acuerdo rápido, utilizando un lenguaje ya existente en el PF-DESC, en lugar de explorar nuevas maneras de garantizar que el nuevo mecanismo se adapte al niño y contemple sus necesidades". Según la red NGO Group, esto provocó que algunos derechos de la CDN fueran pasados por alto (por ejemplo, el interés superior del niño) y que se favoreciera el "mínimo común denominador"32. Una pérdida significativa en términos de opciones procedimentales fue la eliminación del mecanismo de comunicaciones colectivas durante el último día de las negociaciones ${ }^{33}$. Este mecanismo hubiera permitido a las instituciones nacionales de derechos humanos, defensores de los niños y ONGs del Consejo Económico y Social (ECOSOC) presentar comunicaciones alegando violaciones de la CDN. Mientras que ninguno de los otros mecanismos de reclamaciones de Derechos Humanos de las Naciones Unidas establece tal mecanismo, su inclusión en un proceso de reclamaciones específico de niños sin dudas hubiese estado justificado dado el frecuente fracaso de los sistemas legales domésticos para hacer efectiva la capacidad de agencia de los niños y otras obligaciones en términos del artículo 12. Por esta razón, es extremadamente lamentable que haya sido finalmente excluido del Protocolo.

Otro aspecto decepcionante (pero no del todo sorprendente) del PF3-CDN fue la inclusión de un lenguaje tomado del Protocolo Facultativo del Comité de DESC (PF-DESC) para el establecimiento de los criterios de admisibilidad del Procedimiento de Comunicaciones en relación al examen de comunicaciones que denuncien violaciones de derechos económicos, sociales o culturales. El artículo 10(4) del PF-CDN señala que "al examinar una comunicación en que se denuncien violaciones de derechos económicos, sociales o culturales, el Comité considerará hasta qué punto son razonables las medidas adoptadas por el Estado parte de conformidad con el Artículo 4 de la Convención. Al hacerlo, el Comité tendrá presente que el Estado parte puede adoptar toda una serie de posibles medidas de política para hacer efectivos los derechos económicos, sociales y culturales enunciados en la Convención". Esta disposición se basa en el artículo 8(4) PF-DESC, un subartículo que fue incluido como respuesta a los esfuerzos por parte de una serie de países por incorporar un "margen de apreciación" para los Estados en lo relativo a los derechos enunciados en esa Convención, lo cual encontró una gran resistencia de las ONGs. Existe un debate sobre si "lo razonable" será interpretado a los efectos de proporcionar un más amplio "margen de apreciación" para los países en lo que respecta a la implementación de DESC o si incorporará un estándar de revisión para el cumplimiento de los derechos como lo realizado en la jurisprudencia constitucional de Sudáfrica, lo cual supondría una carga significativamente mayor para los Estados $^{34}$. ¿Cómo interpretará el Comité esta disposición? Sólo el tiempo lo dirá.

31 Para mayor información sobre el proceso de redacción del Grupo de Trabajo Abierto sobre el Protocolo Facultativo sobre la Convención de los Derechos del Niño, ver [en línea] <http://www.ohchr.org/EN/HRBodies/HRC/WGCRC/Pages/ OpenEndedWorkingGrouplndex.aspx> [consulta: 15 abril 2013]

32 Para mayor información, ver [en línea] <http://www.childrightsnet.org/docs/FileManager/NGOGroup/HRC17_Item_5_-Joint_oral_statement_on_OP_CRC_-_FINAL.pdf> [consulta: 15 abril 2013].

33 Para mayor información, ver [en línea] <http://www.childrightsnet.org/NGOGroup/childrightsissues/ComplaintsMechanism/> [consulta: 15 de abril 2013].

34 Para una consideración de las diferentes maneras en que el Artículo 8(4) puede ser interpretado, ver MAHON, Claire. "Progress at the Front: The Draft Optional Protocol to the International Covenant on Economic, Social and Cultural Rights". Human Rights Law Review Vol. 8, No. 4, 2008 y PORTER, Bruce. "The Reasonableness of Article 8(4): Adjudicating Claims from the Margins". Nordic Journal of Human Rights, Vol. 27, No. 1 
Quizá la falencia más importante en lo relativo al mecanismo de reclamaciones del PF-CDN en términos del artículo 12 es que fue redactado por adultos. Mientras que las personas con discapacidades jugaron un papel clave en la redacción de la Convención sobre los Derechos de Personas con Discapacidades -poniendo en práctica la consigna "¡nada sobre nosotros sin nosotros!"- los niños no participaron de ninguna manera significativa en la redacción del PF-CDN. Teniendo esto en cuenta, parecería que el artículo 12 sigue siendo ignorado por actores que deberían comportarse de otro modo, en contextos en los que dicho artículo debería jugar un papel importante. De este modo, hasta que todos los responsables en la toma de decisiones -diseñadores de políticas y de leyes, funcionarios públicos, jueces, abogados, defensores de derechos humanos, instituciones nacionales e internacionales de derechos humanos, guardianes y padres- otorguen la atención adecuada a la opinión del niño, la implementación total del artículo 12 de la CDN seguirá siendo un sueño lejano. 
